\title{
Exit-Wave Reconstruction and Phase Analysis Using a Hitachi SU9000 FE-SEM
}

\author{
S. Rudinsky ${ }^{1}$ and R. Gauvin ${ }^{1}$ \\ ${ }^{1 .}$ Department of Mining and Materials Engineering, McGill University, Montreal, Canada
}

With consistently new and improving technologies, transmission imaging of thin specimens is no longer reserved to high-energy conventional transmission electron microscopes (TEM). Low energy bright-field and dark-field imaging can now be performed in field-emission scanning electron microscopes (FE-SEM). This enables the possibility of transmission imaging at accelerating voltages around $30 \mathrm{keV}$ and lower, which can reduce knock-on beam damage of fragile materials. With this, it is of interest to obtain the exit wave function for phase analysis and holography applications [1]. Various different methods for reconstructing the exit wave function from experimental data are currently used, each depending on the application and restrictions of the instrument [2,3]. One possible method for computing the exit wave function is to use iterative wave function reconstruction (IWFR) [4]. With IWFR, a focal series of experimental images is used to obtain the wave function at the exit plane of the specimen. First, an initial guess is made. Then, this guess is used to perform inverse propagation from each image plane to the exit plane. An average is taken over all images and the sum-squared error between the current iteration and the previous one is calculated. A new guess is then made and a subsequent iteration is performed. This procedure is continued until the error between iterations has met some tolerance.

Here, we use a focal series of images of nano-sized copper particles obtained in a Hitachi SU9000 FE-SEM to perform an iterative wave function reconstruction. Because there is no lens system below the specimen, the wave function propagation can be modeled as that of a free particle. The algorithm follows that of previous work performed on soft X-rays where a mixing is done at each image plane before a final wave function is obtained at the exit plane [5]. Determining the phase of the exit wave function can provide various types of information about the instrument. For example, the Hitachi SU9000 has a through-thelens specimen holder which causes a magnetic field to be generated around the specimen. How the wave function is affected by this magnetic field can be investigated through the IWFR technique. Focal series taken of a specimen inside various regions of the lens can be used to reconstruct the wave function under the influence of different field strengths. The computed phases can then be used to determine shifts that may be caused by this magnetic field.

An initial focal series of three dark-field images of copper particles deposited on a carbon grid was taken at a specimen height centered in the middle of the lens. These images were obtained at a defocus of 0 , 1000, and $2000 \mathrm{~nm}$ and are depicted in Fig 1. A cross-correlation was first performed to ensure the images were properly aligned prior to performing the reconstruction. The images were then padded with the average value at the boundaries in order to aid in convergence of the method. The amplitude and phase of the associated exit-wave reconstruction are shown in Fig 2. The heights of each particle in a cluster and their overlap can be distinguished in the phase of the wave function. On the other hand, the amplitude is accurately associated with the experimental image taken at a defocus of $0 \mathrm{~nm}$. This method could be used in conjunction with wave function simulation methods such as Bloch wave to provide more information about thin specimens imaged in the SEM. 
References:

[1] A. Tonomura. "Electron Holography". Springer Series in Optical Sciences. (Springer Berlin, Heidel-berg).

[2] T. Duden et al. Microscopy and Microanalysis 20 (2014), p. 968.

[3] A. Morgan et al. Ultramicroscopy 111 (2011), p. 1455.

[4] L. Allen et al. Ultramicroscopy 100 (2004), p. 91.

[5] L. Allen, W McBride, and M. Oxley. Optics Communications 233 (2004), p. 77.

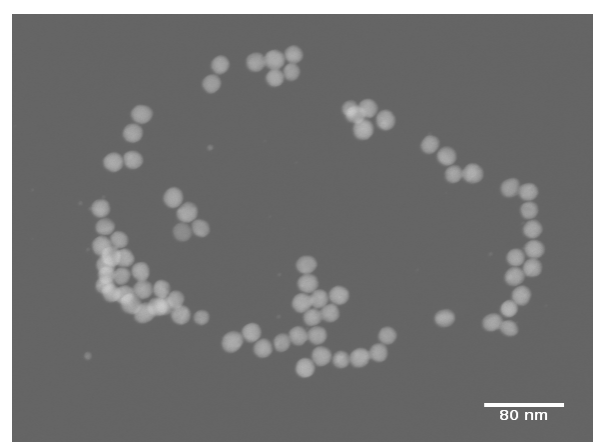

(a)

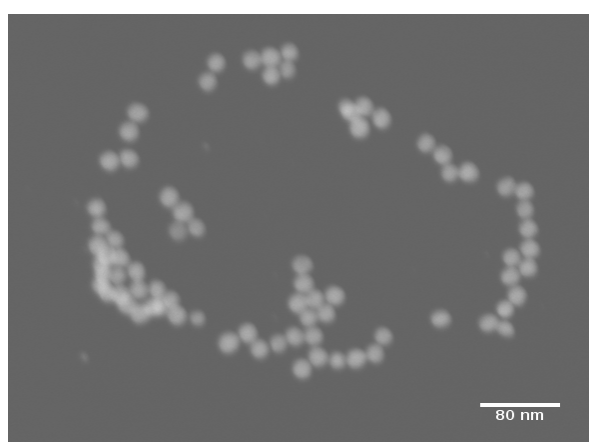

(b)

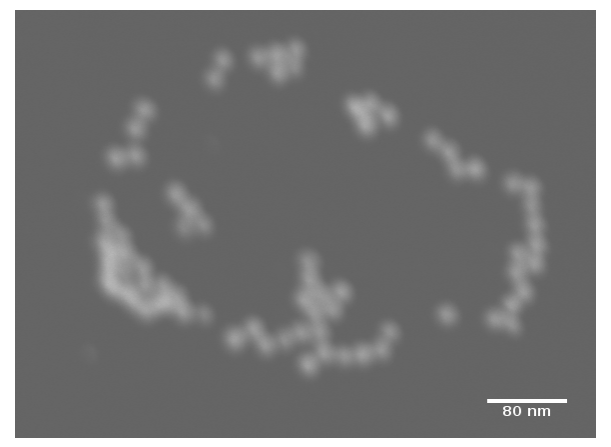

(c)

Figure 1. Dark-field experimental images taken at a defocus of (a) $0 \mathrm{~nm}$, (b) $1000 \mathrm{~nm}$, and (c) $2000 \mathrm{~nm}$.

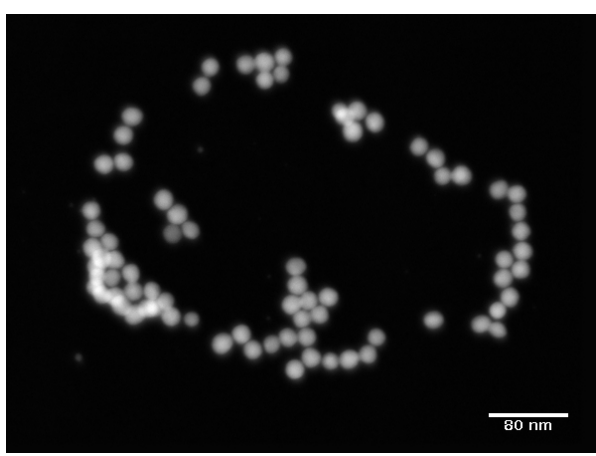

(a)

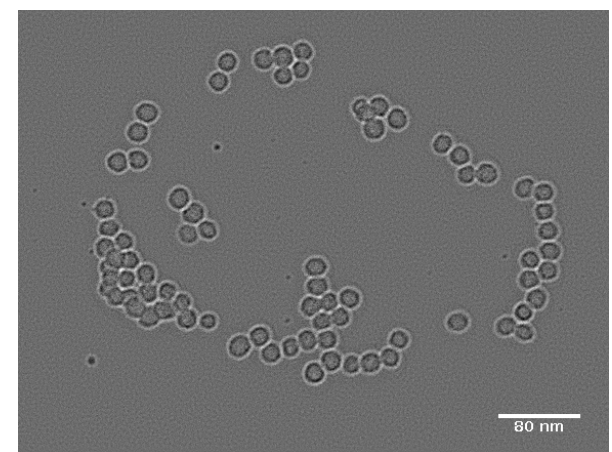

(b)

Figure 2. (a) Amplitude and (b) phase of exit wave function generated by IWFR method. 